\title{
A novel way of therapeutic angiogenesis using an adeno-associated virus-mediated angiogenin gene transfer
}

\author{
Young-Hwa Cho ${ }^{1 *}$, Hyun Park ${ }^{2 *}$, \\ Eui-Sic $\mathrm{Cho}^{3}$, Wun-Jae $\mathrm{Kim}^{4}$, \\ Bong-Su Kang ${ }^{1}$, Byung-Yong Park ${ }^{3}$, \\ Yeun-Ju Kim ${ }^{1}$, Young-III Lee ${ }^{5}$, \\ Soo-lk Chang ${ }^{2,6}$ and Keerang Park ${ }^{1,6}$ \\ ${ }^{1}$ Department of Biotechnology \\ Juseong College \\ Chungbuk 363-794, Korea \\ ${ }^{2}$ Department of Biochemistry \\ Chungbuk National University \\ Cheongju 361-763, Korea \\ ${ }^{3}$ School of Dentistry \\ Chonbuk National University \\ Jeonju 561-756, Korea \\ ${ }^{4}$ College of Medicine and Institute for Tumor Research \\ Chungbuk National University \\ Cheongju 361-763, Korea \\ ${ }^{5}$ School of Engineering \\ University of Suwon \\ Suwon 445-743, Korea \\ ${ }^{6}$ Corresponding authors: Tel, 82-43-210-8462; \\ Fax, 82-43-210-8465; E-mail, krpark@jsc.ac.kr (for K.R.P.) and \\ Tel, 82-43-261-2318; Fax, 82-43-267-2306; \\ E-mail, sichang@cbnu.ac.kr (for S.I.C.) \\ *These authors contributed equally to this work.
}

Accepted 18 April 2007

Abbreviations: AAV, adeno-associated virus; GFP, green fluorescent protein; HUVECs, human umbilical vein endothelial cells; rAAV, recombinant $\mathrm{AAV}$

\footnotetext{
Abstract

To develop a novel therapeutic angiogenesis for the treatment of cardiovascular diseases, angiogenin (ANG1) was examined as a potential therapeutic gene. An adeno-associated virus (AAV)-mediated gene delivery system was used to measure the therapeutic efficacy of ANG1. Using a triple cotransfection technique, rAAV-ANG1-GFP, rAAVVEGF-GFP and rAAV-GFP vectors were produced, which were then used to infect human umbilical vein endothelial cells (HUVECs) in order to evaluate in vitro angiogenic activities. Their protein expressions, tagged with green fluorescent protein (GFP),
}

were monitored by confocal microscopy. The functional activities were measured using woundhealing HUVEC migration assays. The number of migrated cells stimulated by both the expressed ANG1 and the VEGF in rAAV-infected HUVECs increased almost twice the number observed in the expressed GFP control. In vivo angiogenic activities of the expressed ANG1 or VEGF were determined using mouse angiogenesis assays. The angiogenic activities of ANG1 or VEGF expressed in the injected mice were increased by 1.36 and 2.16 times, respectively, compared to those of the expressed GFP control. These results demonstrate that the expressed ANG1 derived from rAAV infection has in vitro and in vivo angiogenic activities and suggest that the rAAV-ANG1 vector is a potential strategy for therapeutic angiogenesis.

Keywords: dependovirus; angiogenesis; angiogenin; cardiovascular disease; gene therapy

\section{Introduction}

New therapeutic approaches using gene transfer of angiogenic regulators have been extensively investigated and applied for the treatment of cardiovascular diseases, such as peripheral arterial disease and ischemic heart disease. They are considered promising strategies for the replacement of current pharmacological intervention and surgical revascularization. Since 1994, when the VEGF was first applied as a treatment for patients with peripheral vascular disease, VEGF and FGF have been most commonly applied to develop angiogenic gene therapy using various delivery vehicles. These studies showed positive effects for the promotion of neo-vascularisation (Kastrup, 2003; Rissanen et al., 2003; Kim et al., 2004; Lee et al., 2005). However, VEGF has mostly been either associated with stimulated angiogenesis in tumors, production of nonfunctional leaky vessels or used for enhancement of atherosclerotic plaque development through an increase in focal macrophage levels. So the notion of angiogenic therapy using VEGF as a therapeutic gene remains a somewhat controversial proposal (Springer et al., 1998; Celletti et al., 2001).

Angiogenin (ANG1) is one of the most potent inducers of new blood vessel formation. It is normally present in plasma and interacts with endo- 
thelial and smooth muscle cells to induce a wide range of cellular responses, including cell migration and proliferation and formation of tubular structures. Angiogenin is the only angiogenic factor known to exhibit ribonucleolytic activity and belongs to the pancreatic RNase superfamily of proteins (Pavlov and Badet, 2001). However, angiogenin as a potential therapeutic gene has not been tested.

In the present study, we investigated both in vitro and in vivo therapeutic efficacies of the ANG1 gene using an AAV-mediated gene delivery system by measuring angiogenic activities of the expressed ANG1. The AAV, as one of the ideal gene delivery vectors, was employed due to prominent features such as non-pathogenic properties, prolonged expression and low immunogenicity. Protein expressions in the transduced HUVECs by rAAV-ANG1GFP, rAAV-VEGF-GFP, or rAAV-GFP were verified by a confocal microscopy. In vitro and in vivo functional activities of the expressed ANG1 and VEGF were examined using wound healing HUVEC migration assays as well as mouse angiogenesis assays.

\section{Materials and Methods}

\section{Plasmid construction}

Total RNA was prepared from bovine liver using TRIzoL reagent (Invitrogen Life Technologies, Inc., Carlsbad, CA). RNA was reverse-transcribed to cDNA using oligo (dT) and random hexamer primers according to the manufacturer's protocol (1st Strand cDNA Synthesis Kit for RT-PCR, Roche Diagnostics Corp., Basel, Switzerland). The first strand of cDNA was then used in subsequent RT-PCR reactions to amplify the open reading frame of the ANG1 (bovine nt 231-674, accession number AF135124) flanked by $K p n l$ and Apal for a pAAV-ANG1-GFP construct with the ANG1-specific primers (F1 sense primer, 5'-ggggtaccgccaccATGGTCATGGTCCTGAG-3'; R1 non-sense primer for GFP-tagged ANG1 construct, 5'-ccgggcccgGTGGCGTGGAGTGATAAAG-3'). For comparison of therapeutic efficacy, pAAV-VEGFGFP and pAAV-VEGF constructs were established as follows: VEGF cDNA (human nt 1039-1683, accession number NM_003376) was amplified using VEGF-specific primers (VEGF-A sense primer, 5'ggggtaccgccaccATGAACTTTCTGCTGTCT-3'; VEGF-A non-sense primer 1 for pAAV-VEGF-GFP, 5'-ttgggeccGCCTCGGCTTGTCACATCT-3'; VEGF-A non-sense primer 2 for pAAV-VEGF, 5'-ccctcgagTCACCGCCTCGGCTTGT-3'). The lower case letters indicate the addition of restriction endonuclease sites to the 5' and 3' ends of the PCR products. To make a GFP-tagged ANG1 or VEGF construct, we first made a pAAV-GFP construct by inserting GFP
cDNA from pEGFP-N1 (Clontech, Mountain View, $C A$ ) into the linearized $p A A V$ derived from $p A A V-F I X$ (Herzog et al., 1997). Both of the amplified ANG1 or VEGF cDNA was digested by Kpnl and Apal and subcloned into the linearized pAAV-GFP either to make pAAV-ANG1-GFP or to construct pAAVVEGF-GFP. The other PCR products of VEGF digested by $\mathrm{Kphl}$ and $\mathrm{Xhol}$ were placed into the linearized $p A A V$ prepared from $p A A V-F I X$ to construct pAAV-VEGF. All pAAV plasmids contain the cytomegalovirus (CMV) promoter/enhancer and the coding sequence for either the ANG1 gene (444 bp) or the VEGF (645 bp), either alone or fused to the $\mathrm{N}$-terminus of GFP, and the simian virus (SV) 40 polyadenylation signal flanked by the AAV serotype 2 (AAV2) inverted terminal repeat sequences. All subclones were verified by restriction enzyme analyses followed by sequencing to confirm GFP fusion constructs in frame.

\section{Recombinant AAV (rAAV) vector production and purification}

The triple co-transfection and purification protocols were employed for rAAV vector production with minor modifications (Matsushita et al., 1998). HEK293 cells were maintained as described previously (Park et al., 2001) and transiently co-transfected with the following three plasmids using the calcium phosphate methods: (i) a pAAV plasmid containing an expression cassette for the ANG1, VEGF or GFP cDNA under CMV promoter/enhancers (pAAV-ANG1GFP, pAAV-VEGF-GFP, pAAV-VEGF or pAAV-GFP, respectively), (ii) an adenoviral helper plasmid (pHelper plasmid, Stratagene, La Jolla, CA), (iii) an AAV helper plasmid encoding AAV serotype 2 (AAV2) rep functions and AAV2 cap genes (pAAV$\mathrm{RC}$, Stratagene). The rAAV serotype 2 (rAAV2) vectors were purified by repeated $\mathrm{CsCl}$ gradient centrifugation and produced yields in excess of the titer of $10^{12}$ viral genomes (v.g.)/ml as determined by a quantitative slot blot hybridization.

\section{Localization of the protein expression in HUVECs transduced with rAAV vectors}

HUVECs (Clonetics, Cat\# cc-2517, Cambrex Bio Science Walkersville, Inc., Baltimore, MD) were maintained in a complete medium (EGM-2 BulletKit, Cat\# CC-3162, Cambrex Bio Science Walkersville, Inc.) and seeded in six-well plates at a density of 2.0 $\times 10^{5}$ cells per well. The next day they were infected with $4.0 \times 10^{10}$ rAAV vectors and the cells were changed with fresh, complete medium $24 \mathrm{~h}$ later. The transduced HUVECs were mounted on a confocal microscopy to visualize the protein ex- 
pression $48 \mathrm{~h}$ post-infection. GFP was excited with a $488 \mathrm{~nm}$ wavelength and the emitted fluorescence was monitored at $>500 \mathrm{~nm}$.

\section{Wound healing HUVEC migration assay}

In vitro angiogenic activities of the expressed ANG1 and VEGF were measured using a wound-healing HUVEC migration assay. HUVECs were seeded in six-well plates at a density of $2.0 \times 10^{5}$ cells and infected individually with $4.0 \times 10^{10} \mathrm{v}$.g. of three different rAAV vectors for $24 \mathrm{~h}$. The cells were then wounded with a cell scraper and changed with an EBM-2 medium containing 1\% FBS (starvation medium, Cat\# CC-3156, Cambrex Bio Science Walkersville, Inc., Baltimore, MD). Cell migration of the samples was continuously monitored for $24 \mathrm{~h}$ using Fluorescence Microscopy (Leica DM IRB Fluorescence Microscope, Germany), and the migrated HUVECs from the wounded positions were counted per field.

\section{Mouse angiogenesis assay (Matrigel assay for angiogenesis)}

All animal experiments were performed in accor- dance with the Chungbuk National University Ethical Procedures for Laboratory Animals, 2004 (Approval Number 2004-76). C57BL/6N mice (7 week-old, male) were injected subcutaneously midway on the right and left ventral sides with a mixture of Matrigel (0.6 ml, BD Biosciences, Bedford, MA), each of three different rAAV vectors $\left(1.0 \times 10^{12}\right.$ v.g. $)$, and heparin $(64 \mathrm{U} / \mathrm{ml}$, Sigma Chemical Co., St. Louis, $\mathrm{MO}$ ). For the positive control, $250 \mathrm{ng}$ bFGF (Invitrogen) were included in the Matrigel mixture instead of rAAV vectors (Senger et al., 1997). After ten days, the mice were euthanized and the implants were isolated to be fixed overnight in Bouin's fixatives, which were then embedded in paraffin. Sections were cut to a thickness of $5 \mu \mathrm{m}$, deparaffinized and stained with a mixture of hematoxylin and eosin. The migration distance of fibrovascular ingrowth into the Matrigel was measured in each of 25 randomly chosen cross-sections per each group using digitized representative photographic images with an analysis program 3.0 (Soft Imaging System, Munster, Germany). The photographs were obtained from three random specimens of each group. Data were described as the average distance \pm standard errors ( $n=25$ for each group). To verify statistical
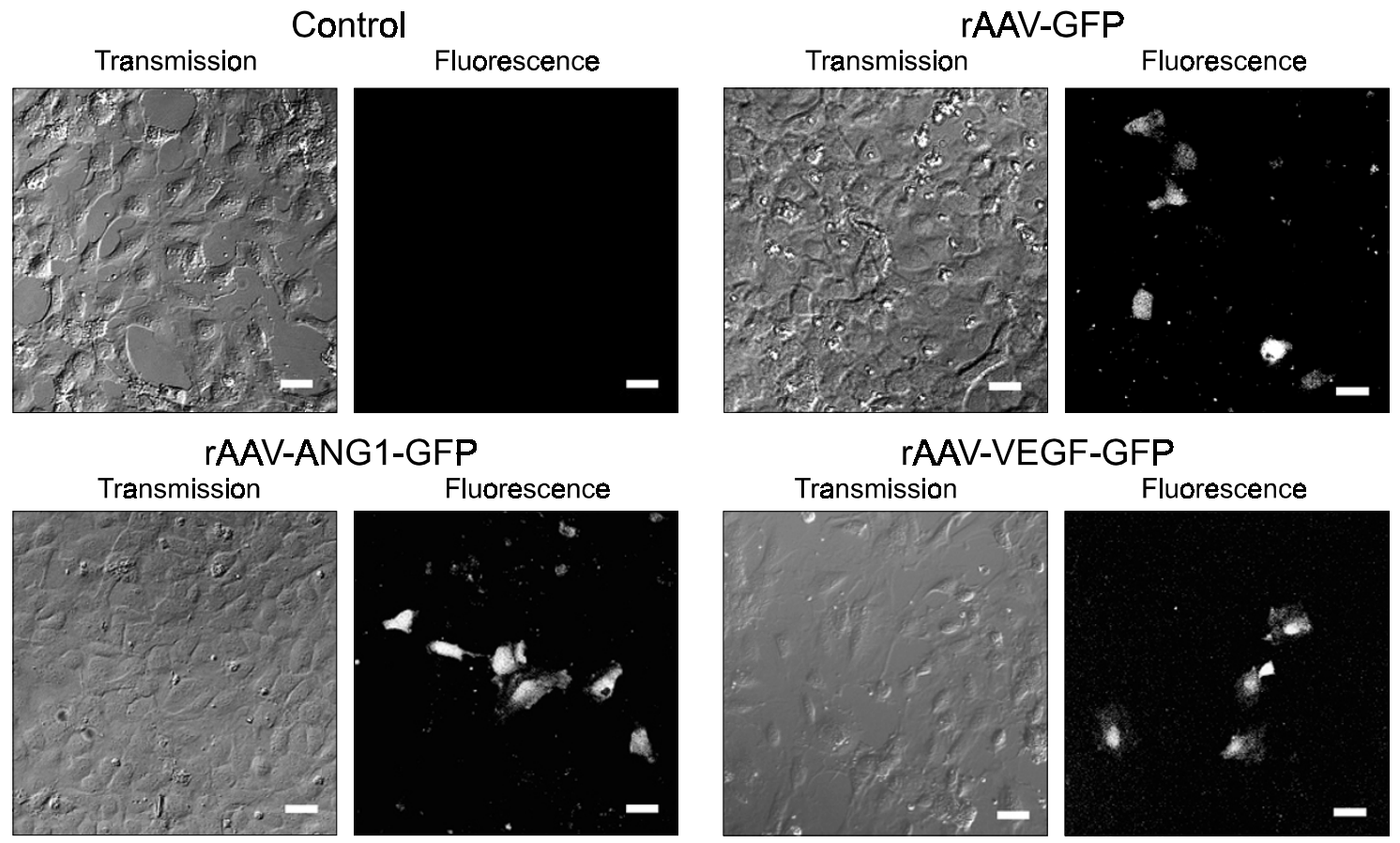

Figure 1. Localization of the expressed GFP-tagged fusion proteins in rAAV vector-transduced HUVECs. HUVECs were infected with three different rAAV vectors, rAAV-ANG1-GFP, rAAV-VEGF-GFP, or rAAV-GFP (200,000 virus genomes (v.g.) per cell) for $24 \mathrm{~h}$ and refed with fresh medium. Forty-eight $h$ later the transduced cells were mounted on the stage and the protein expressions were visualized by a confocal microscopy. The top/left Control panel illustrates the images of the negative control (HEPES), the top/right rAAV-GFP panel illustrates the images for the expressed GFP, the bottom/left rAAV-ANG1-GFP illustrates the images for the expressed ANG1-GFP and the bottom/right rAAV-VEGF-GFP illustrates the images for the expressed VEGF-GFP. The expressed GFP in the cells was excited with a $488 \mathrm{~nm}$ wavelength and the emitted fluorescence was monitored at $>500 \mathrm{~nm}$. The scale bar indicates $40 \mu \mathrm{m}$. 
significance of the obtained results, the data were subjected to the one-way ANOVA using a Tuckey test.

\section{Results}

\section{Preparing an AAV-mediated ANG1 or VEGF gene delivery system}

To demonstrate the therapeutic benefit of a novel angiogenesis gene for patients with cardiovascular disease, particularly ischemia, who may not be adequately treated with available therapies, we prepared an AAV-mediated ANG1 gene transfer system (rAAV-ANG1-GFP) by making a GFP-tagged ANG1 plasmid construct of pAAV-ANG1-GFP as described in Materials and Methods. We also subcloned pAAV-VEGF-GFP and pAAV-VEGF to produce VEGFexpressing AAV vectors (rAAV-VEGF-GFP and rAAV-VEGF, respectively) for comparisons of thera- peutic efficacy. GFP-tagging to a protein has often been used to facilitate monitoring of its expression. These plasmid constructs were separately included for a triple co-transfection with pAAV-RC and pHelper (Stratagene) to produce rAAV vectors.

\section{Monitoring protein expression in HUVECs transduced with rAAV vectors}

To verify that the infected $\mathrm{AAAV}$ vectors may direct the protein expressions of ANG1 and VEGF, we monitored the GFP expression in HUVECs transduced with three different $\mathrm{rAAV}$ vectors 48 -h postinfection using a confocal microscopy. The rAAV vectors were produced and purified by repeated $\mathrm{CsCl}$ gradient centrifugation and produced yields in excess of the titer of $10^{12} \mathrm{v} . \mathrm{g} . / \mathrm{ml}$. As shown in Figure 1, ANG1 and VEGF were mainly localized to the cytoplasmic area, which was similar to the expression profile in HUVECs infected by rAAV-GFP.

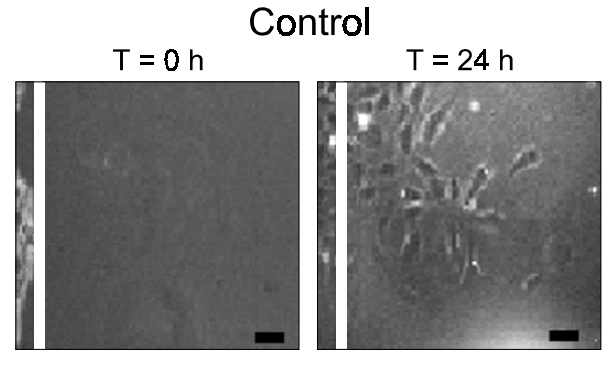

rAAV-ANG1-GFP

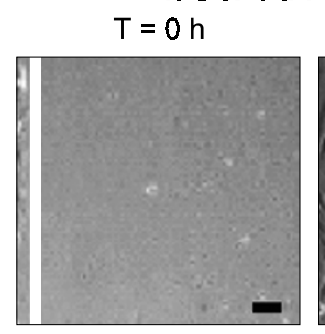

$\mathrm{T}=24 \mathrm{~h}$
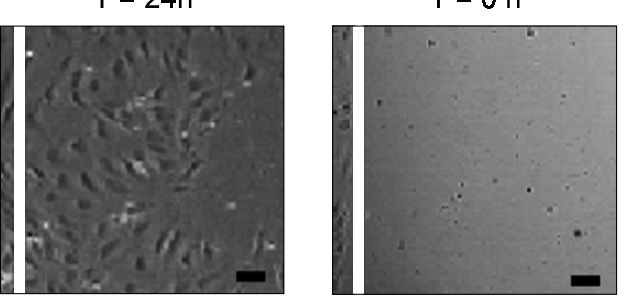

rAAV-VEGF-GFP

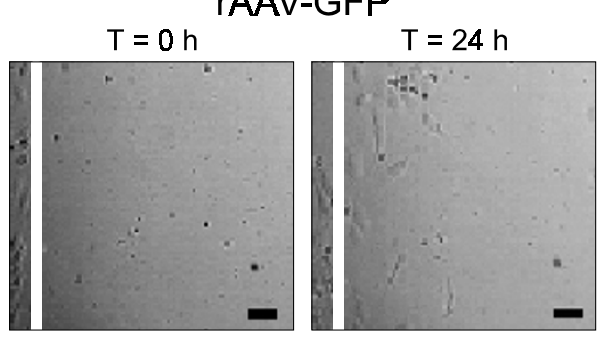

$\mathrm{T}=24 \mathrm{~h}$
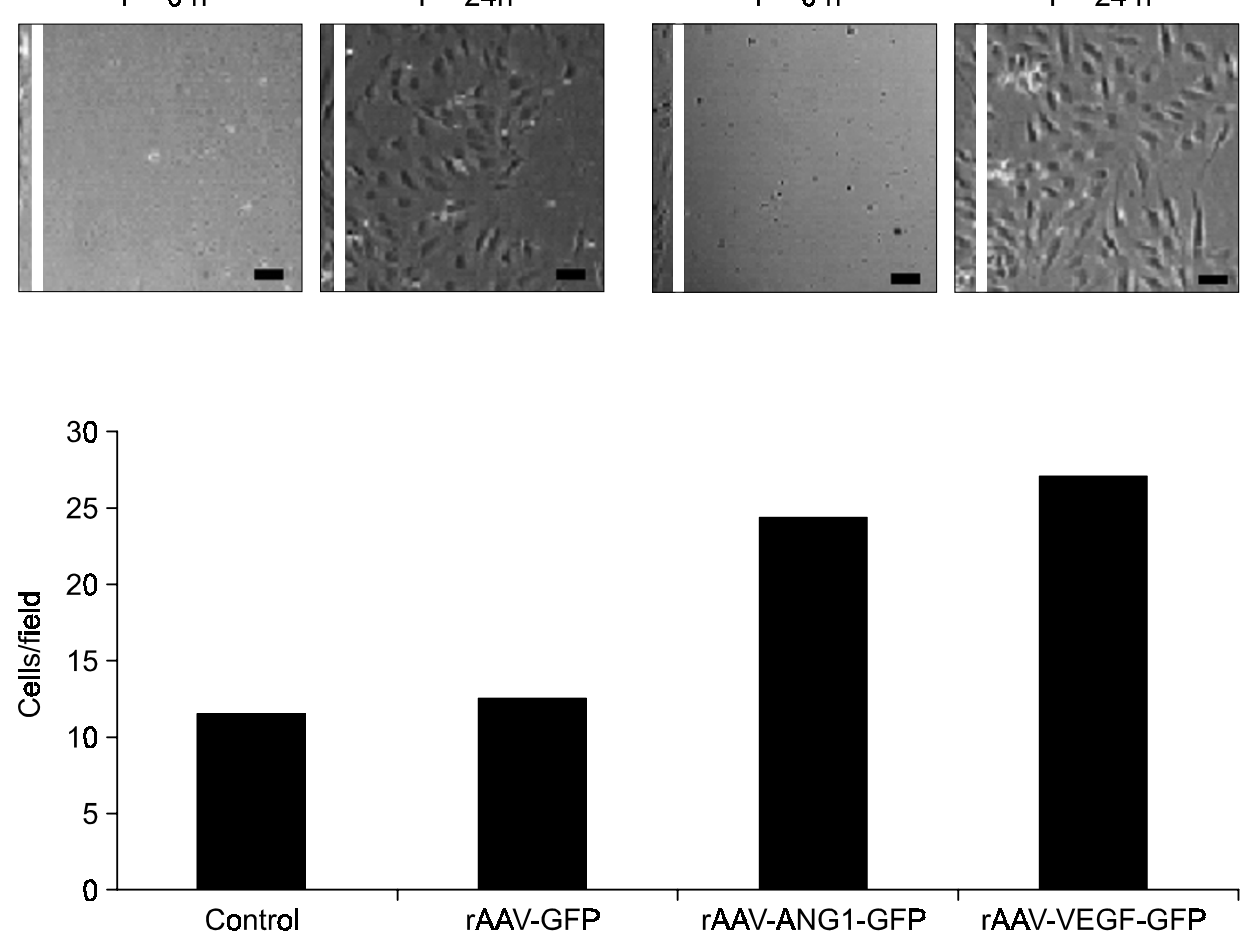

Figure 2. In vitro functional activities for angiogenesis using woundhealing HUVEC migration assays. HUVECs were infected with a HEPES buffer (control), rAAV-GFP, rAAV-ANG1-GFP or rAAV-VEGF for $24 \mathrm{~h}$, re-fed with fresh medium and were wounded using a cell scraper. The cells were mounted on the stage of the fluorescent microscopy and the cell migration of each sample was continuously monitored for $24 \mathrm{~h}$. The other samples were then analyzed individually and monitored accordingly. The images on the top 4 panels were taken at the beginning of monitoring $(T=0 \mathrm{~h})$ and at the end of monitoring ( $T=24 \mathrm{~h}$ ) using a Fluorescence Microscopy (Leica DM IRB Fluorescence Microscope, Germany). The migrated cells per field were counted and plotted in the bottom graph. The experiment was repeated ten times and these results were the representative data. The scale bar indicates $40 \mu \mathrm{m}$. 
The secretion profiles of ANG1 and VEGF from the transduced HUVECs were also examined using western blot analyses with an anti-GFP polyclonal antibody (Clontech). Upon infection of HUVECs with rAAV vectors, the cultured medium was collected every $24 \mathrm{~h}$ to $96 \mathrm{~h}$ and immunoprecipitated with the anti-GFP antibody. Those immunoprecipitates were subjected to western blot analyses using the anti-GFP antibody, but the protein secretion level was not high enough to be detected (data not shown).

\section{Functional angiogenic activities of the expressed ANG1 and VEGF using wound healing HUVEC migration assays and mouse angiogenesis assays}

To measure in vitro angiogenic activities of the expressed ANG1 and VEGF, we performed woundhealing HUVEC migration assays using a fluorescent microscopy (Leica DM IRB Fluorescence Microscope), because angiogenesis often involves endothelial cell migration and proliferation within a collagen-rich extracellular matrix. As shown in Figure 2, the number of migrated HUVECs infected with rAAV-ANG1-GFP or rAAV-VEGF-GFP increased between 1.93 and 2.10 times compared to the
rAAV-GFP control. On the other hand, the number of migrated cells infected with rAAV-GFP was similar to that treated with HEPES as a negative control

To evaluate in vivo angiogenic activities of the expressed ANG1 and VEGF, we measured the distances of fibrovascular ingrowth into the Matrigel. As shown in Figure 3, the degree of fibrovascular ingrowth was 1.36, 2.16 and 2.87 times higher in the Matrigel implants containing rAAV-ANG1-GFP, rAAVVEGF and the purified bFGF (250 ng, Invitrogen), respectively, in comparison with fibrovascular ingrowth into the Matrigel containing rAAV-GFP.

\section{Discussion}

The purpose of this study was to establish a safe and effective gene therapeutic angiogenesis using the seldom-investigated AAV-mediated angiogenin (ANG1) gene transfer system, rAAV-ANG1 (Kastrup, 2003; Rissanen et al., 2003; Kim et al., 2004; Lee et al., 2005). We selected ANG1 as a potential therapeutic gene and AAV as a safer and effective gene delivery vector because of the following characteristics. ANG1 is a potent inducer of neovascularization that interacts with endothelial and
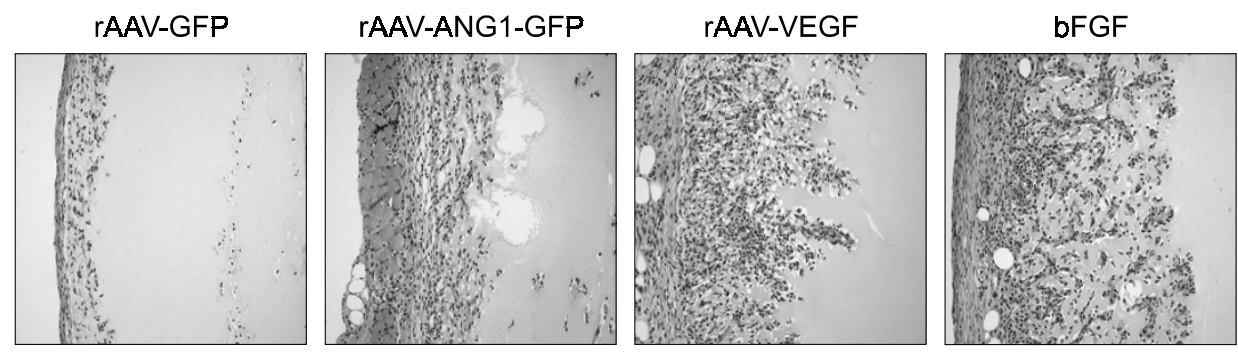

Figure 3. In vivo functional activities for angiogenesis using a mouse angiogenesis assay. Matrigel containing rAAV-GFP, rAAVANG1-GFP, rAAV-VEGF or a purified bFGF (250 ng, Invitrogen) were injected into mice. Ten days

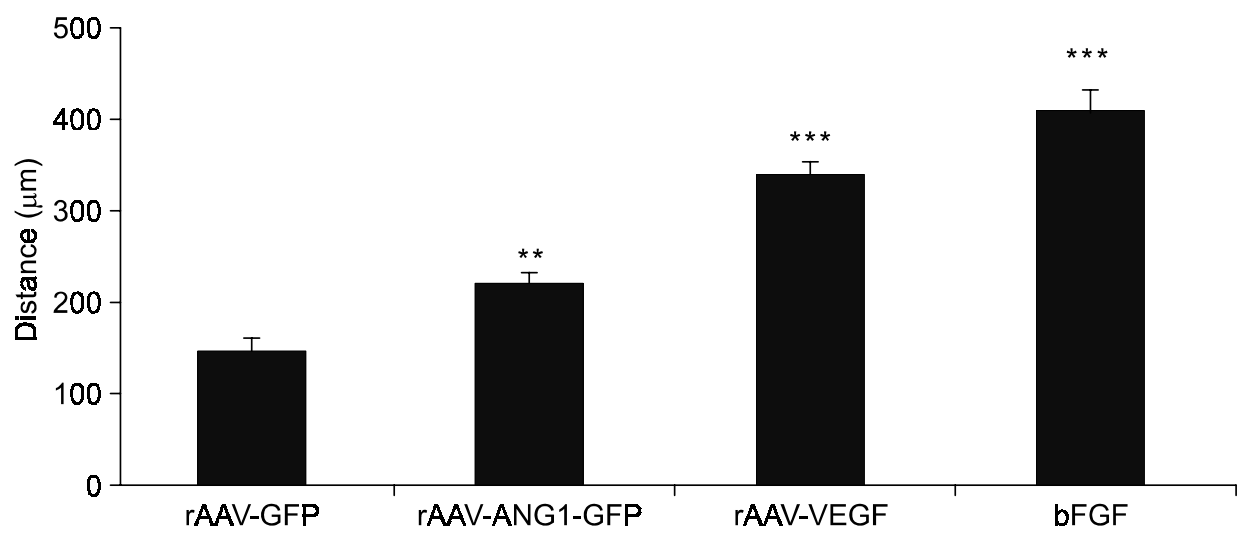
later the mice were euthanized and the Matrigel implants were isolated to determine the activities of newly forming microvessels. The top panels are four pictures of the H\&E staining processed from the isolated Matrigel as described in Materials and Methods. The migrated distances of the fibrovascular ingrowth into the Matrigel were measured from 25 sections per each group in a random manner and plotted in the bottom graph $(n=25)$. Data in the bottom table are mean \pm SE obtained from the experiments $(P<0.05)$. To verify statistical significance of the obtained results, the data were subjected to the one-way ANOVA using a Tuckey test.

\begin{tabular}{|c|c|c|c|c|}
\hline rAAV/protein & rAAV-GFP & rAAV-ANG1-GFP & rAAV-VEGF & bFGF \\
\hline Distance $(\mu \mathrm{m})$ & $156.4 \pm 16.24$ & $212.46 \pm 10.26$ & $338.52 \pm 13.25$ & $408.79 \pm 22.61$ \\
\hline
\end{tabular}

**:P<0.05,***:P<0.001 
smooth muscle cells to induce cell migration, proliferation, and formation of tubular structures. ANG1, however, has not been tested as an angiogenesis therapy (Strydom, 1998; Pavlov and Badet, 2001). Among viral gene delivery systems, such as retrovirus, adenovirus, AAV, and lentivirus, AAV is regarded as one of the best if the size of the therapeutic gene is small enough for rAAV to be packaged efficiently (Lundstrom, 2003). It is nonpathogenic and elicits no inflammatory responses. The AAV vector shows long-term transgene expression and it is able to transduce both dividing and non-dividing cells, such as endothelial cells, skeletal muscles, cardiac myocytes, neurons, lungs, hepatocytes, and renal cells (Herzog et al., 1997; Rabinowitz and Samulski, 1998; Lu, 2004).

We utilized the GFP-tagging constructs to facilitate localization of the expressed proteins directed by rAAV infections and we were able to localize the GFP-tagged ANG1 and VEGF protein expressions in the rAAV-transduced HUVECs using a confocal microscopy (Figure 1). However, probably due to a low transduction efficiency of AAV2 into HUVECs (Hsieh et al., 2005), we could not quantitatively determine protein expression in the infected cell lysates nor in the culture medium using western blot analyses with the anti-GFP antibody (Clontech). Nonetheless, we were able to measure the angiogenic activities of the expressed ANG1 and VEGF in the rAAV-infected HUVECs, whereas the expressed GFP derived from rAAV infection showed no such angiogenic activity, as shown in Figure 2. When HUVECs were transduced with rAAV-ANG1-GFP or rAAV-VEGF-GFP vectors, the number of migrated cells increased similarly by 1.93 and 2.10 times, respectively, compared to those infected with rAAVGFP, and also to those treated with HEPES.

The results obtained from the mouse angiogenesis assay were slightly different from those of the wound-healing HUVEC migration assay. In the mouse angiogenesis assay, we included rAAV-VEGF instead of rAAV-VEGF-GFP in the injected Matrigel. Of particular note were the migrated distances of the fibrovascular ingrowth into the Matrigel containing rAAV-VEGF, which was 1.59 times further than those with rAAV-ANG1-GFP. This result allowed us to speculate that GFP as a cytosolic protein tagging to ANG1 might have inhibited secretion in the expressed protein. Therefore, the expressed VEGF directed by rAAV-VEGF injection could have been secreted better than the expressed ANG1 by rAAV-ANG1-GFP administration, which resulted in higher in vivo angiogenic activities in rAAV-VEGF injected mice than for those in rAAV-ANG1-GFP administered mice. This speculation can be supported by our recent study of the angiogenic gene therapy using a naked DNA system (submitted to Cardiovascular Research). In the HUVEC migration assay, the number of the migrated cells transfected by pVEGF-GFP plasmid construct was similar to that of pANG1-GFP naked DNA, whereas the migrated distances of fibrovascular ingrowth into the Matrigel containing pVEGF-GFP was about the same as that containing pANG1-GFP in the mouse angiogenesis assay (data not shown). However, we can not rule out the possibility of differences between in vivo assay systems and in vitro assay systems.

In summary, this study demonstrated that AAVmediated ANG1 gene transfer stimulated in vitro and in vivo angiogenic activities that induced the HUVECs' migration and fibrovascular ingrowth. These findings suggest that rAAV-ANG1 can be an effective, and novel, angiogenic therapeutic vector that may be further developed for treatment of cardiovascular diseases.

\section{Acknowledgement}

We thank Dr. Katherine A. High for providing an AAV vector production system and the pseudotyped serotypes. We also thank Drs. Heesoon Chang, Roland Herzog and Mee-Young Ahn for technical assistance during the study. This work was supported by a grant from the Korea Science and Engineering Foundation (KOSEF) of R01- 2002-000-00539-0, and partly supported by the grant from the Ministry of Science and Technology (M10534040005-05N3404-00511), Seoul, Korea. This work was also partly supported by the research grant of the Chungbuk National University in 2005.

\section{References}

Celletti FL, Waugh JM, Amabile PG, Brendolan A, Hilfiker PR, Dake MD. Vascular endothelial growth factor enhances atherosclerotic plaque progression. Nat Med 2001;7:425-9

Herzog RW, Hagstrom JN, Kung SH, Tai SJ, Wilson JM, Fisher KJ, High KA. Stable gene transfer and expression of human blood coagulation factor IX after intramuscular injection of recombinant adeno-associated virus. Proc Natl Acad Sci USA 1997; $94: 5804-9$

Hsieh MY, Liu YL, Schlachterman A, High KA. AAV-mediated gene transfer to endothelial cells. Mol Ther 2005;11:S153-4

Kastrup J. Therapeutic angiogenesis in ischemic heart disease: gene or recombinant vascular growth factor protein therapy? Curr Gene Ther 2003;3:197-206

Kim HJ, Jang SY, Park JI, Byun J, Kim DI, Do YS, Kim JM, Kim S, Kim BM, Kim WB, Kim DK. Vascular endothelial growth factor-induced angiogenic gene therapy in patients with peripheral artery disease. Exp Mol Med 2004;36:336-44

Lee JS, Byun J, Kim JM, Kim CY, Kim BM, Chung JH, Jang Y, Kim DK. Cardiac expression profiles of the naked DNA vectors 
encoding vascular endothelial growth factor and basic fibroblast growth factor. Exp Mol Med 2005;37:447-56

Lu Y. Recombinant adeno-associated virus as delivery vector for gene therapy-A review. Stem Cells Dev 2004;13:133-45

Lundstrom K. Latest development in viral vectors for gene therapy. Trends Biotechnol 2003;21:117-22

Matsushita T, Elliger S, Elliger C, Podsakoff G, Villarreal L, Kurtzman GJ, Iwaki Y, Colosi P. Adeno-associated virus vectors can be efficiently produced without helper virus. Gene Ther 1998;5:938-45

Park K, Begenisich T, Melvin JE. Protein kinase A activation phosphorylates the rat $\mathrm{CIC}-2 \mathrm{Cl}$ - channel but does not change activity. J Membr Biol 2001;182:31-7

Pavlov N, Badet J. Angiogenin: involvement in angiogenesis and tumor growth. Bull Cancer 2001;88:725-32

Rabinowitz JE, Samulski J. Adeno-associated virus expre- ssion systems for gene transfer. Curr Opin Biotechnol 1998;9:470-5

Rissanen TT, Markkanen JE, Arve K, Rutanen J, Kettunen MI, Vajanto I, Jauhiainen S, Cashion L, Gruchala M, Narvanen O, Taipale P, Kauppinen RA, Rubanyi GM, YlaHerttuala S. Fibroblast growth factor 4 induces vascular permeability, angiogenesis, and arteriogenesis in a rabbit hindlimb ischemia model. FASEB J 2003;17:100-2

Senger DR, Claffey KP, Benes JE, Perruzzi CA, Sergiou AP, Detmar M. Angiogenesis promoted by vascular endothelial growth factor: regulation through $\alpha 1 \beta 1$ and $\alpha 2 \beta 1$ integrins. Proc Natl Acad Sci USA 1997;94:13612-7

Springer ML, Chen AS, Kraft PE, Bednarski M, Blau HM. VEGF gene delivery to muscle: potential role for vasculogenesis in adults. Mol Cell 1998;2:549-58

Strydom DJ. The angiogenins. Cell Mol Life Sci 1998;54: 811-24 